\title{
Desarrollo de un Trabajo de Fin de Grado Multidisciplinar en Ingeniería Civil como preparación del alumno para la vida profesional
}

\author{
Francisco Javier Camacho Torregrosa ${ }^{a}$, Ana María Pérez Zuriagab, Hugo Coll \\ Carrilloc, Julián Alcalá González ${ }^{d}$, Inmaculada Romero Gil ${ }^{\mathrm{e}}$ y María Elvira Garrido de \\ la Torref \\ aUniversitat Politècnica de València, fracator@tra.upv.es, bUniversitat Politècnica de València,

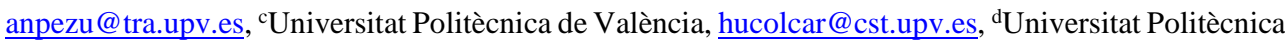 \\ de València, jualgon@cst.upv.es, eUniversitat Politècnica de València, inrogi@dihma.upv.es, \\ fUniversitat Politècnica de València, egarrido@trr.upv.es.
}

\begin{abstract}
This paper covers the inception and development of a new type of Final Degree Project carried out in the Civil Engineering School. More than 20 students collaborate in this Project, which is a major innovation over previous ones. This Project consists on the development of three alternatives for the CV-190 road diversión in Figueroles (Castellón). This kind of project allows the students to reach a deep knowledge on their skills, as well as they are encouraged to collaborate. Some advantages are presented, as well as some drawbacks that should be resolved for further editions.
\end{abstract}

Keywords: teamworking, collaboration, Final Degree Project, Civil Engineering, Public Works Engineering, road, multidisciplinary

\section{Resumen}

En el presente documento se presenta el planteamiento y desarrollo de un Trabajo Fin de Grado realizado en la Escuela Técnica Superior de Ingenieros de Caminos, Canales y Puertos. La novedad de dicho trabajo consiste en la cooperación masiva de más de 20 alumnos con el fin de conseguir tres diferentes soluciones alternativas a la carretera CV-190 en el término municipal de Figueroles (Castellón). Este tipo de proyecto permite que los alumnos alcancen un elevado grado de especialización, a la vez que fomenta la colaboración entre ellos. Se presentan las ventajas observadas, así como los inconvenientes que sería razonable solventar en futuras ediciones de este tipo de trabajo. 
Desarrollo de un Trabajo de Fin de Grado Multidisciplinar en Ingeniería Civil como preparación del alumno para la vida profesional

Palabras clave: trabajo en equipo, colaboración, Trabajo Fin de Grado, Ingeniería Civil, Ingeniería de Obras Públicas, carretera, multidisciplinar

\section{Introducción}

El actual Espacio Europeo de Educacion Superior (conocido como "Plan Bolonia”) ha supuesto un importante cambio en los estudios universitarios. Parte de ello es la realización del Trabajo Final de Grado (TFG), que sustituye la función del anterior Proyecto Final de Carrera (PFC). El RD 1393/2007 y su modificación RD 861/2010 disponen que, con carácter general, todos los títulos oficiales “concluirán con la elaboración y defensa” de un Trabajo Fin de Grado (TFG) o Trabajo Fin de Máster (TFM), según el caso.

Los anteriores PFC presentaban una heterogeneidad importante dentro de las diferentes universidades y, en particular, en la Universitat Politècnica de València. Particularizando en la Escuela Técnica Superior de Ingenieros de Caminos, Canales y Puertos (ETSICCP), los PFC podían ser de tipo I (proyecto constructivo convencional) o tipo II (proyecto de otro tipo o proyecto parcial). En cualquier caso, los créditos correspondientes eran 4,5 para las cuatro titulaciones de la Escuela (las tres de Ingeniero Técnico de Obras Públicas y la de Ingeniero de Caminos, Canales y Puertos). El desarrollo se realizaba de forma individual por el alumno, así como su defensa, que tenía lugar en sesión pública frente a un Tribunal conformado por profesores de la Escuela.

Con el objetivo de adaptar de forma homogénea los criterios de desarrollo y exposición del TFG en todas las titulaciones de la Universidad, el 7 de marzo de 2013 se aprobó en Consejo de Gobierno de la UPV la Normativa Marco de Trabajos Fin de Grado y Fin de Máster. Uno de los puntos más importantes implica la orientación de los grados hacia el ejercicio profesional, mientras que los másteres son enseñanzas más avanzadas que pueden estar dirigidas hacia la especialización profesional o la iniciación en el campo de la investigación. Fruto de ello, se establece que los TFG deberán consistir en trabajos profesionales.

Otro de los puntos tratados por dicha Normativa Marco es la duración de los trabajos. Por diversas causas, los anteriores PFC por lo general se dilataban en exceso en el tiempo, suponiendo trabajos de mucha mayor carga que los que deberían corresponder a los créditos asignados. Por ello, en la Normativa se encomienda a las Entidades Responsables de Títulos (ERT) a corregir esta desviación, y a fomentar que los trabajos se desarrollen en un plazo de tiempo razonable (implicando por tanto una densidad del trabajo también razonable). En muchos casos, el periodo de tiempo está limitado por la continuidad natural de los alumnos a cursar el Máster que sucede al Grado, lo que acaba siendo un plazo de entre tres y seis meses. En particular, en la ETSICCP se dotó tanto al Grado de Ingeniería de Obras Públicas 
(GIOP) como al Grado en Ingeniería Civil (GIC) de 12 créditos ECTS, equivalentes a entre 300 y 360 horas de trabajo del alumno.

Con el fin de compatibilizar adecuadamente el desarrollo del TFG con las asignaturas del último curso, la Normativa Marco incluso sugiere la posibilidad de organizar el TFG "como si de una asignatura más se tratase, mediante actividades docentes regladas con asistencia del estudiante a los correspondientes seminarios, talleres o cualquier otra manera de organizar la docencia para esta actividad”.

Una de las partes más novedosas de la Normativa Marco estriba en el desarrollo de los trabajos. En el punto 4 del artículo 3 establece que "la originalidad del trabajo [...] debe entenderse sin menoscabo de que pueda ser parte independiente e individual de un trabajo integral desarrollado de manera conjunta entre estudiantes de una misma titulación o de diferentes titulaciones y ERT. En cualquier caso, la defensa del TFG y TFM debe ser individual”. Esto implica que los alumnos pueden realizar un mismo trabajo de forma conjunta, pudiendo centrarse cada uno con mayor detalle en partes separadas del mismo, fomentando de este modo el trabajo en equipo (dimensión competencial DC6 "trabajo en equipo y liderazgo"), la cual será determinante en su vida profesional.

Los Trabajos Fin de Grado comenzaron a realizarse en las titulaciones de GIC y GIOP en el curso 2013/2014. Varios de los autores de este documento fueron tutores de TFG, observando algunas limitaciones y potencialidades. Al tratarse de un trabajo desarrollado en equipo, puede darse pie a que los alumnos trabajen de forma similar a una empresa de consultoría. Esto les permitiría profundizar en aspectos organizativos, de liderazgo y de trabajo en equipo, lo que convertiría el TFG en el nexo de unión entre las enseñanzas teóricas de la carrera con el mundo profesional. Por otra parte, un enfoque multidisciplinar del alumnado implica la necesidad de que los tutores sean también especialistas en diversas disciplinas.

Un aspecto de suma importancia a la hora de realizar trabajos en equipo consiste en la similitud de metas u objetivos. Cuantos más alumnos existan en el grupo, más heterogéneo será, y por tanto más probabilidades de que exista un fallo. Un trabajo multidisciplinar funciona como una cadena, en la que cada alumno es un eslabón. El fallo de uno de ellos podría implicar el fallo en la secuencia del conjunto, perjudicando seriamente. Esto obliga a formular con cuidado la metodología de trabajo.

\section{Objetivos}

En el presente curso 2014/2015, ocho profesores de diferentes disciplinas con experiencia previa en PFCs y TFGs en la ETSICCP decidieron conformar un equipo con el fin de dirigir un proyecto multidisciplinar a un gran número de alumnos. La idea principal de este trabajo

(cc)) EY-NC-ND 2015, Universitat Politècnica de València

Congreso In-Red (2015) 
Desarrollo de un Trabajo de Fin de Grado Multidisciplinar en Ingeniería Civil como preparación del alumno para la vida profesional

es permitir que los alumnos se desarrollen de forma proactiva en dirección al mundo profesional. Esto conlleva que a lo largo del proyecto se lleven a cabo:

- Seminarios para la enseñanza de las herramientas de trabajo profesionales, particularizados para cada una de las especialidades contempladas.

- Tutorías individuales y en grupo para solventar las dudas que vayan surgiendo a lo largo del desarrollo.

- Talleres de trabajo en grupo, asistidos por los profesores, en los que todos los alumnos compartirán la información y el desarrollo del trabajo.

En todo momento, se busca que los profesores presenten un papel dual: mostrar la información inicial para que los alumnos puedan ponerse rápidamente a trabajar, y guiar para que sean ellos mismos quienes, de forma proactiva, puedan alcanzar la solución al problema planteado.

La presente comunicación pretende mostrar el planteamiento de este esquema de trabajo, haciendo especial hincapié en la organización del mismo. También se valorará, con la experiencia de este curso, los aspectos que es necesario corregir de cara a futuras ediciones. Debido a que se trata del primer curso, todavía no se dispone de resultados oficiales, pero se indicará qué resultados se esperan con la máxima fiabilidad posible.

\section{Desarrollo de la innovación}

\subsection{Organización inicial}

\subsubsection{Conformación del equipo}

El equipo fue conformado por ocho profesores de diferentes áreas de conocimiento dentro de la ingeniería civil. En aquellas materias más relevantes para el TFG conjunto, son dos los profesores que participan, con el fin de dar un mejor servicio a los alumnos, pues el número de los mismos será mayor.

Los profesores, indicando su disciplina, son los que aparecen en la Tabla 1.

Tabla 1. Profesores tutores del TFG Multidisciplinar

\begin{tabular}{lcc}
\hline Profesor & $\begin{array}{c}\text { Área de } \\
\text { conocimiento }\end{array}$ & Categoría \\
\hline Alcalá González, Julián & Estructuras & Profesor Contratado Doctor \\
Albentosa Hernández, Eduardo & Hidráulica & $\begin{array}{c}\text { Profesor Titular Escuela } \\
\text { Universitaria }\end{array}$ \\
\hline
\end{tabular}


F.J. Camacho Torregrosa, A.M. Pérez Zuriaga, H. Coll Carrillo, J. Alcalá González, I. Romero Gil y M.E. Garrido de la Torre

\begin{tabular}{lcc}
\hline $\begin{array}{l}\text { Camacho Torregrosa, Francisco } \\
\text { Javier }\end{array}$ & Carreteras & Profesor Ayudante \\
Coll Carrillo, Hugo & Estructuras & Profesor Asociado \\
Garrido de la Torre, María Elvira & Geotecnia & $\begin{array}{c}\text { Profesor Titular Escuela } \\
\text { Universitaria }\end{array}$ \\
Pérez Zuriaga, Ana María & Carreteras & Profesor Ayudante Doctor \\
Romero Gil, Inmaculada & Medio & Profesor Titular de Universidad \\
Vallés Morán, Francisco José & Ambiente & Profesor Titular Escuela \\
& Hidráulica & Universitaria \\
\hline
\end{tabular}

\subsubsection{Presentación del proyecto y distribución inicial de alumnos}

El proyecto planteado consiste en desarrollar una nueva variante que circunde la carretera CV-190 a su paso por Figueroles. Existen diversas posibilidades para esta nueva variante: norte, centro y sur (Figura 1). Aunque no existe mucho tráfico (por lo que no se justifica el proyecto por funcionalidad), la actual travesía presenta un alto volumen de vehículos pesados, siendo la sección transversal muy reducida en diversos puntos. Todo ello ocasiona importantes inconvenientes a los vecinos.

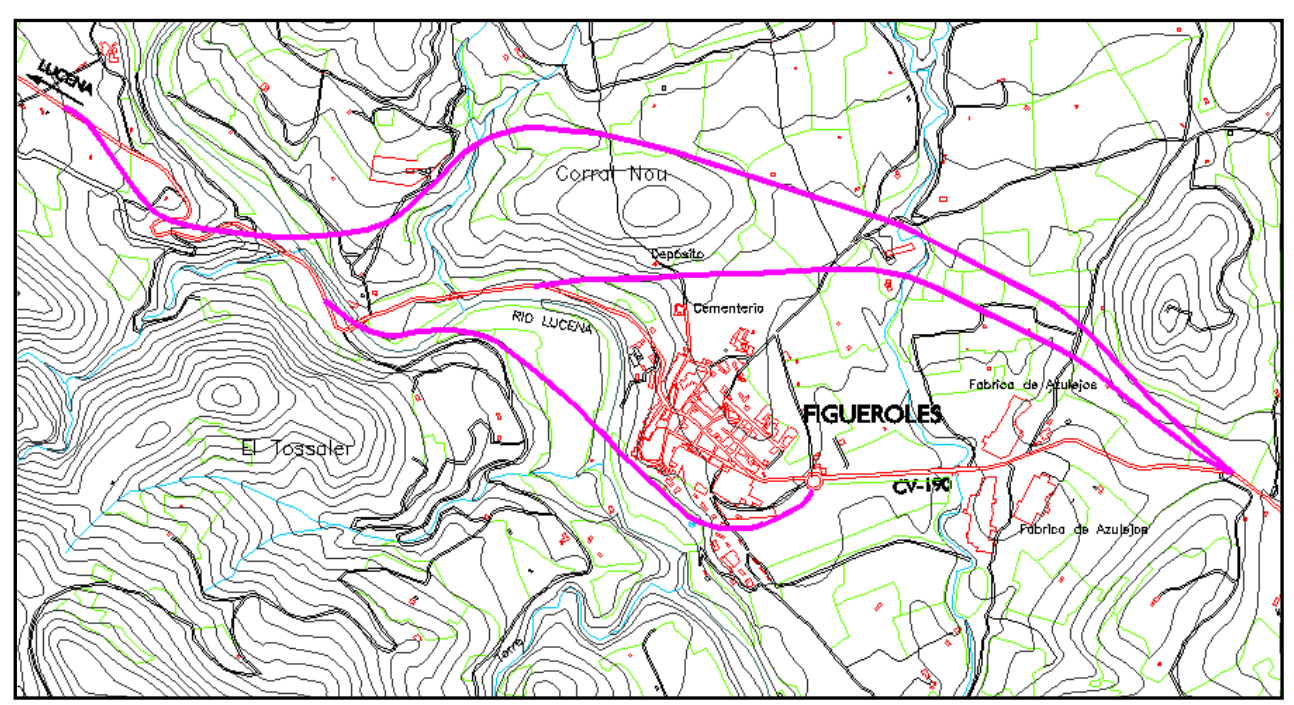

Figura 1. Esquema inicial de las variantes al municipio de Figueroles.

\section{(cc) EY-NC-ND 2015, Universitat Politècnica de València}

Congreso In-Red (2015) 
Desarrollo de un Trabajo de Fin de Grado Multidisciplinar en Ingeniería Civil como preparación del alumno para la vida profesional

Se pretende que en el TFG Multidisciplinar se abarque el estudio pormenorizado de cada una de estas variantes, a nivel de Proyecto Básico. El esquema de la Figura 1 responde a un boceto inicial, no correspondiéndose con el trazado finalmente desarrollado por las alternativas.

Cada una de las alternativas lleva trabajos de trazado, firmes, estudio de tráfico, diseño y dimensionamiento de puentes y obras de fábrica, estudio hidrológico y drenaje, así como estudio hidráulico de cada uno de los puentes.

Tabla 2. Oferta inicial de trabajos

\begin{tabular}{|c|c|c|}
\hline \multicolumn{3}{|c|}{ TFGs para cada una de las tres alternativas } \\
\hline Título & Disciplina & Profesores tutores \\
\hline $\begin{array}{l}\text { Concepción estructural y diseño de } \\
\text { tableros de los puentes }\end{array}$ & \multirow{3}{*}{ Estructuras } & \multirow{3}{*}{$\begin{array}{l}\text { Julián Alcalá González y } \\
\text { Hugo Coll Carrillo }\end{array}$} \\
\hline $\begin{array}{l}\text { Concepción estructural y diseño de } \\
\text { soportes y cimentaciones }\end{array}$ & & \\
\hline $\begin{array}{l}\text { Concepción estructural y diseño de las } \\
\text { obras de fábrica }\end{array}$ & & \\
\hline Diseño geométrico y del firme & \multirow{3}{*}{ Carreteras } & \multirow{3}{*}{$\begin{array}{c}\text { Francisco Javier Camacho } \\
\text { Torregrosa y Ana María } \\
\text { Pérez Zuriaga }\end{array}$} \\
\hline Análisis del tráfico y de la seguridad vial & & \\
\hline Diseño de los nudos & & \\
\hline Estudio hidrológico y drenaje transversal & \multirow[b]{2}{*}{ Hidráulica } & \multirow[b]{2}{*}{$\begin{array}{l}\text { Eduardo Albentosa } \\
\text { Hernández y Francisco } \\
\text { José Vallés Morán }\end{array}$} \\
\hline $\begin{array}{l}\text { Diseño hidráulico de las obras de } \\
\text { ingeniería fluvial para la protección del } \\
\text { puente \#i* }\end{array}$ & & \\
\hline \multicolumn{3}{|c|}{ TFGs globales } \\
\hline $\begin{array}{l}\text { Análisis de la situación actual y propuesta } \\
\text { de mejoras }\end{array}$ & Carreteras & $\begin{array}{c}\text { Francisco Javier Camacho } \\
\text { Torregrosa y Ana María } \\
\text { Pérez Zuriaga }\end{array}$ \\
\hline $\begin{array}{l}\text { Estudio de Impacto Ambiental en todos los } \\
\text { corredores. Análisis sobre el medio físico } \\
\text { Estudio de Impacto Ambiental en todos los } \\
\text { corredores. Análisis sobre el medio biótico }\end{array}$ & $\begin{array}{c}\text { Medio } \\
\text { Ambiente }\end{array}$ & Inmaculada Romero Gil \\
\hline Estudio geológico-geotécnico & Geotecnia & $\begin{array}{c}\text { María Elvira Garrido de la } \\
\text { Torre }\end{array}$ \\
\hline $\begin{array}{r}\text { *: Este TFG se realiza para cada obra de } \\
\text { desde } 1\end{array}$ & $\begin{array}{l}\text { Iso que teng } \\
\text { ista } 2 .\end{array}$ & a alternativa, pudiendo ir \\
\hline
\end{tabular}

Por otra parte, hay una serie de trabajos comunes a todas las alternativas, como son el estudio de impacto ambiental, el estudio geológico-geotécnico, o el análisis de la alternativa 0 (sin 
construcción de variante). Estos TFG también se ofertan y trabajarán conjuntamente con todas las alternativas.

Así pues, el planteamiento inicial de distribución de trabajos es el que aparece en la Tabla 2. Es importante remarcar que se trata únicamente de un planteamiento inicial, pues su distribución final dependerá del número y características de los alumnos que finalmente se interesen por la realización del trabajo. El número final de trabajos ofertados fue, por tanto, de 31.

\subsubsection{Búsqueda y elección de alumnos}

En la ETSICCP existen actualmente dos modalidades para la asignación del TFG a los alumnos: subasta y concierto directo.

La subasta consiste en la propuesta por un profesor o grupo de profesores de un título de TFG, indicando en qué consiste, así como el número de alumnos que lo desarrollarán conjuntamente (o si se trata de un TFG individual). Todos los títulos a subastar son indicados a la Escuela al inicio del curso. Los alumnos que eligen la modalidad de subasta escogen entre los títulos ofertados, asignándoseles por orden de expediente. De esta forma, los alumnos tienen la seguridad de que tendrán asignado un TFG, pero el inconveniente, tanto para ellos como para los profesores, de que el resultado no puede ser reajustado (por ejemplo: dos alumnos no pueden decidir que irán juntos en un TFG).

La asignación de TFGs mediante el concierto directo es similar al procedimiento habitual para los antiguos PFCs: alumno (o grupo de alumnos) y profesor llegan a un acuerdo sobre el TFG. De este modo, los alumnos que deseen trabajar conjuntamente pueden establecer los grupos a su voluntad, hablando posteriormente con el profesor para acordar el TFG. En este punto radica precisamente la ventaja del método: ambas partes disponen de toda la información a la hora de cerrar el acuerdo de colaboración. El posible inconveniente estriba en que el alumno tiene que buscar un profesor que esté dispuesto a tutorizarle, no teniendo la seguridad de éxito.

El grupo de profesores estimó conveniente acogerse a la segunda modalidad (concierto directo), ya que, al tratar con tantos alumnos, prefirió tener un control sobre los alumnos que finalmente se acogían al mismo. El objetivo era minimizar los posibles problemas de compenetración entre ellos, dando facilidad a los alumnos que venían con grupos previamente formados.

Los profesores publicaron un anuncio en los diferentes departamentos con la información del TFG, así como la dirección de correo electrónico a la que debían dirigirse en caso de estar interesados. La oferta estaba abierta tanto a alumnos individuales como a grupos, solicitándose la lista de preferencias entre los proyectos, así como el expediente académico. Para la formación de grupos y el envío del email se dio un plazo de unas dos semanas. Con

(cc)) EY-NC-ND 2015, Universitat Politècnica de València

Congreso In-Red (2015) 
Desarrollo de un Trabajo de Fin de Grado Multidisciplinar en Ingeniería Civil como preparación del alumno para la vida profesional

el fin de dar la máxima difusión posible, también se solicitó a la Escuela que distribuyese el anuncio entre todos los alumnos de último curso.

Se recibieron un total de 28 solicitudes. Para asociar los alumnos a los trabajos individuales, se siguió el siguiente orden de prioridades:

- Mantener juntos a los alumnos que habían solicitado el trabajo de forma conjunta. De este modo se parte de su conocimiento previo como base para una resolución de conflictos más rápida.

- Agrupar a los alumnos según similitud de expedientes. En principio, alumnos con número similar de asignaturas pendientes y/o similar nota de expediente tenderán a compartir similitudes en el proceso de afrontar el trabajo.

Este proceso tuvo lugar a lo largo de las dos primeras semanas de octubre. Tras este proceso, a cuatro alumnos no se les asignó ningún trabajo puesto que era incompatible con sus preferencias. Teniendo en cuenta que el proceso de subasta ya había sido realizado, estos alumnos fueron asignados a otros TFGs dirigidos por algunos de los profesores, pero fuera ya del ámbito de este trabajo.

Igualmente, algunos de los trabajos quedaron vacantes tras el proceso, por lo que hubo que reformular algunos títulos. A lo largo del desarrollo del TFG también se observó que a raíz de los resultados, algunos de los trabajos no tendrían prácticamente profundidad, por lo que se reformularon con tiempo suficiente y procurando afectar lo mínimo posible a los alumnos afectados. También en las fases tempranas de desarrollo se incorporaron algunos alumnos nuevos para cubrir huecos vacantes anteriormente. Finalmente, el número de TFGs individuales dependientes de este trabajo fueron 25 , con los títulos detallados en la Tabla 3 .

Tabla 3. Distribución final de trabajos presentados

Título general: Concurso para el Proyecto de Construcción de la variante CV-190 a su paso por el municipio de Figueroles (provincia de Castellón).

\begin{tabular}{lll}
\hline Alternativa & Subtítulo & Especialidad \\
\hline & Diseño y dimensionamiento del puente & Estructuras \\
& sobre el barranco al Este de Figueroles & \\
\cline { 2 - 3 } Alternativa Norte & Diseño y dimensionamiento del puente & Estructuras \\
& sobre el río Lucena & \\
\cline { 2 - 3 } & Diseño geométrico y del firme & Carreteras \\
\cline { 2 - 3 } & Análisis del tráfico y de la seguridad vial & Carreteras \\
\cline { 2 - 3 } & Diseño de los nudos & Carreteras \\
\cline { 2 - 3 } & Estudio hidrológico y drenaje transversal & Hidráulica \\
\hline Alternativa & Diseño y dimensionamiento del puente & Estructuras \\
Centro & sobre el río Lucena & \\
\hline
\end{tabular}

(c)) EY-NC-ND 2015, Universitat Politècnica de València 


\begin{tabular}{|c|c|c|}
\hline & $\begin{array}{l}\text { Diseño y dimensionamiento del puente } \\
\text { sobre el barranco al Este de Figuroles }\end{array}$ & Estructuras \\
\hline & Diseño geométrico y del firme & Carreteras \\
\hline & Análisis del tráfico y de la seguridad vial & Carreteras \\
\hline & Diseño de los nudos & Carreteras \\
\hline & Estudio hidrológico y drenaje transversal & Hidráulica \\
\hline & $\begin{array}{l}\text { Diseño hidráulico de las obras de ingeniería } \\
\text { fluvial para la protección del puente sobre } \\
\text { el río Lucena }\end{array}$ & Hidráulica \\
\hline \multirow{8}{*}{ Alternativa Sur } & $\begin{array}{l}\text { Concepción estructural y diseño de tableros } \\
\text { del puente sobre el río Lucena }\end{array}$ & Estructuras \\
\hline & $\begin{array}{l}\text { Concepción estructural y diseño de } \\
\text { subestructuras y obras de fábrica }\end{array}$ & Estructuras \\
\hline & $\begin{array}{l}\text { Concepción estructural y diseño del tablero } \\
\text { del puente sobre el barranco del Tossal de } \\
\text { la Negra }\end{array}$ & Estructuras \\
\hline & Diseño geométrico y del firme & Carreteras \\
\hline & Análisis del tráfico y de la seguridad vial & Carreteras \\
\hline & Diseño de los nudos & Carreteras \\
\hline & Estudio hidrológico y drenaje transversal & Hidráulica \\
\hline & $\begin{array}{l}\text { Diseño hidráulico de las obras de ingeniería } \\
\text { fluvial para la protección del puente sobre } \\
\text { el río Lucena }\end{array}$ & Hidráulica \\
\hline \multirow{4}{*}{ General } & $\begin{array}{l}\text { Análisis de la situación actual y propuesta } \\
\text { de mejoras }\end{array}$ & Carreteras \\
\hline & $\begin{array}{l}\text { Estudio de Impacto Ambiental en todos los } \\
\text { corredores. Análisis sobre el medio físico }\end{array}$ & Medio Ambiente \\
\hline & $\begin{array}{l}\text { Estudio de Impacto Ambiental en todos los } \\
\text { corredores. Análisis sobre el medio biótico }\end{array}$ & Medio Ambiente \\
\hline & Estudio geológico-geotécnico & Geotecnia \\
\hline
\end{tabular}

\subsection{Esquema de seminarios y talleres}

Durante el primer semestre del último curso de las titulaciones de GIC y GIOP los alumnos cursan la mayoría de las últimas asignaturas de su titulación. De este modo, el segundo semestre de dicho curso está prácticamente libre para poder concentrarse en el estudio de idiomas y el desarrollo del TFG. Por el motivo anterior, el desarrollo de trabajos y talleres se llevó a cabo a partir de enero de 2015.

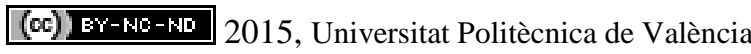

Congreso In-Red (2015) 
Desarrollo de un Trabajo de Fin de Grado Multidisciplinar en Ingeniería Civil como preparación del alumno para la vida profesional

A finales de octubre de 2014 se realizó una reunión informativa con todos los alumnos, en la que se informó de las asignaciones finales de TFG, así como de la mecánica de funcionamiento. Además, se abrió un espacio en PoliformaT para que los profesores y los alumnos pudieran colgar la información relevante.

La interacción profesor-alumno se ha planteado fundamentalmente a través de tres modalidades diferenciadas: seminarios, trabajo en taller y tutorías.

Los seminarios son clases magistrales y prácticas informáticas en las que el profesor imparte a los alumnos cómo se realiza cierta parte del trabajo. Un ejemplo consiste en aprender a manejar cierta herramienta informática, o indicar cómo se extraen y procesan datos. Estas sesiones se han organizado en función de las necesidades detectadas por los profesores de cada modalidad. El número de seminarios difiere entre modalidades, así como su distribución temporal. En principio, es obligatoria la asistencia a los alumnos de la modalidad correspondiente, siendo voluntaria para el resto.

Los primeros seminarios de cada modalidad sirven para establecer de dónde puede extraerse la información de partida, así como para el primer contacto con las herramientas informáticas. Por ejemplo, en los dos primeros seminarios de carreteras se les indicó a los alumnos cómo descargar la información cartográfica del PNOA (Plan Nacional de Ortofotografía Aérea), o el tráfico. En estructuras se les indicó qué normativa debían conocer, y en hidráulica se les completó la información adquirida durante el grado sobre la delimitación de cuencas hidrológicas y simulación de tormentas.

El trabajo en taller consiste en sesiones periódicas de duración variable (entre dos y cinco horas) en las que los alumnos se reúnen por alternativas y deciden la solución conjunta. Estas sesiones tienen especial relevancia en los periodos inicial y medio del desarrollo del trabajo, cuando los alumnos deben interactuar para ofrecer las diferentes restricciones y posibilidades al resultado final. De esta forma, se configura una solución con la colaboración de todos, e igualmente todos ellos conocen los motivos que les han llevado a la misma.

Estos trabajos en taller se configuraron con una periodicidad semanal, y fueron fijados de acuerdo con todos los alumnos, el viernes de 12 a $17 \mathrm{~h}$. Dichas reuniones fueron asistidas regularmente por los profesores de los diferentes campos, con el fin de resolver dudas que únicamente con la experiencia pueden abordarse (Figura 2).

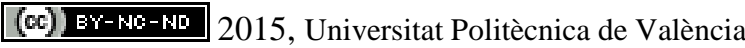

Congreso IN-RED (2015) 


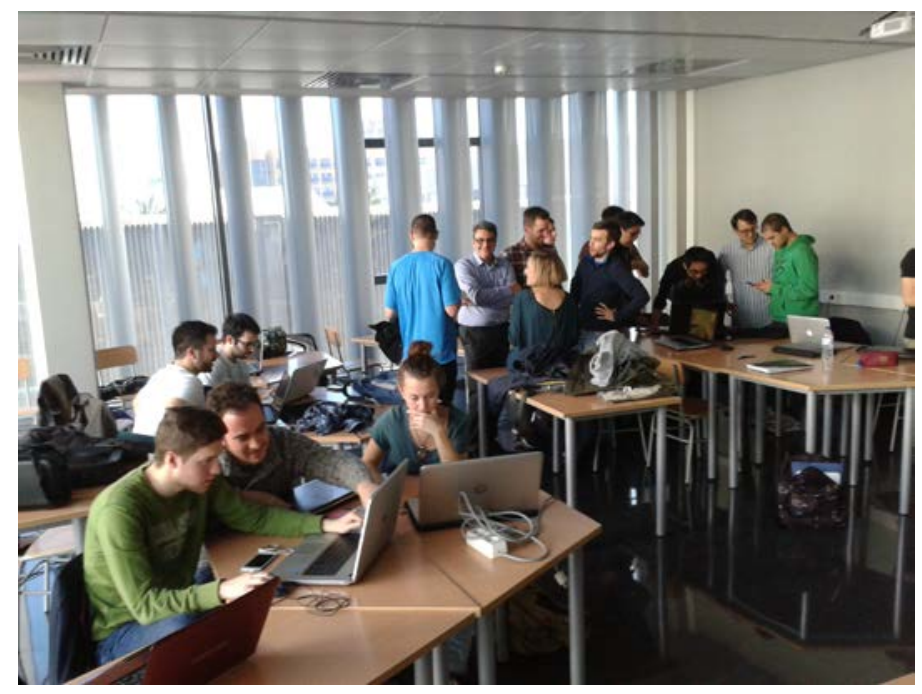

Figura 2. Alumnos y profesores en el taller.

Además de las anteriores sesiones, se hace necesario dedicar un tiempo a tutorías, tanto individuales como en grupos reducidos, donde se abordan temas de complejidad algo superior, particulares de cada tipo de trabajo. Estas tutorías se reservan a petición de los alumnos, como cualquier otra.

\subsection{Visita al lugar de la obra}

Una parte fundamental en la redacción de cualquier proyecto de ingeniería civil consiste en la visita al lugar de la obra. Esta visita permite recoger datos (como el tráfico in situ, materiales, fotografías, etc.), además de disponer de la visión que únicamente la visita al emplazamiento puede ofrecer.

Por ello, a mediados de enero se organizó una visita de campo abierta a todos los alumnos y profesores del TFG. La Escuela colaboró aportando el autobús para el viaje. El momento de la visita de obra tiene que estar bien escogido, pues los alumnos deben estar libres de otros compromisos (exámenes o entregas de trabajos), así como disponer de la primera información (tráfico y un primer vistazo a la zona desde el ordenador). La visita fue preparada mediante una reunión en la que se les indicó en qué cosas debían fijarse, así como los métodos de toma de datos que se iban a seguir.

Durante la visita de campo, se dividió al grupo en dos. El primero realizó una primera inspección por la zona, recogiendo datos geológicos, geotécnicos, hidrológicos, de caminos existentes, líneas eléctricas, etc (Figura 3). El segundo grupo se dividió en las dos entradas del pueblo y tomaron aforos de tráfico. A media mañana, los dos grupos intercambiaron sus roles. 
Desarrollo de un Trabajo de Fin de Grado Multidisciplinar en Ingeniería Civil como preparación del alumno para la vida profesional

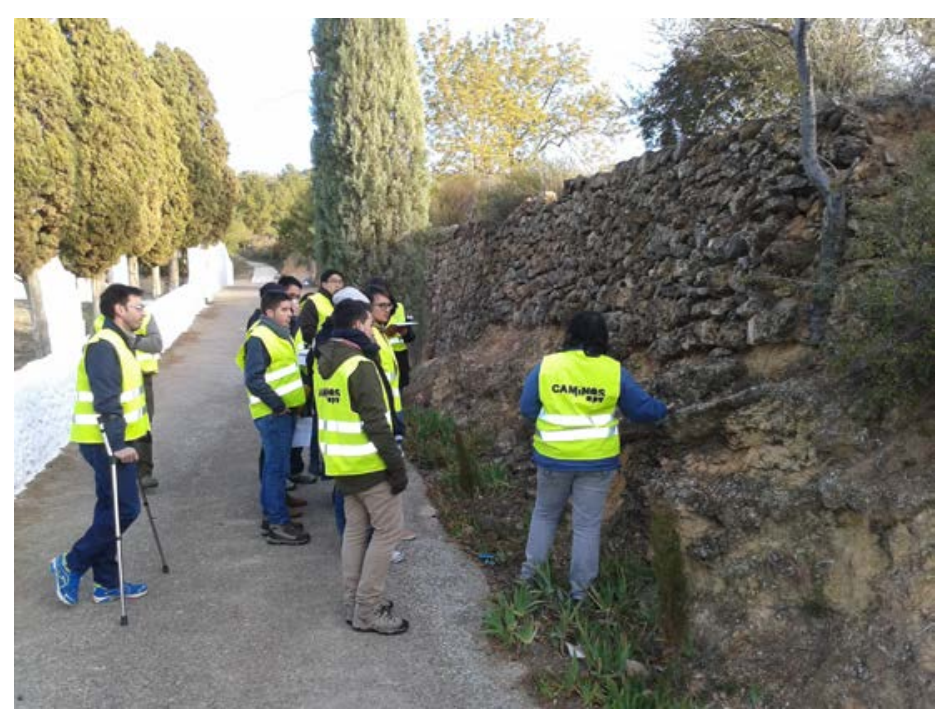

Figura 3. Explicación durante la visita a obra.

\section{Resultados}

En el momento de la redacción del presente documento no se dispone de resultados concluyentes sobre el desarrollo del TFG multidisciplinar, ya que la fecha de entrega de los trabajos está establecida para el 12 de junio. Sin embargo, sí que se disponen de herramientas sufientes para comentar las principales debilidades y fortalezas encontradas y proponer actuaciones para la mejora de los desarrollos de TFG multidisciplinares en el futuro.

\subsection{Debilidades}

La idea con la que se ha propuesto y desarrollado este trabajo consiste en que los alumnos aprendan a trabajar dentro de un equipo multidisciplinar con interacciones entre todos los miembros del equipo, como se trabaja en una consultoría de ingeniería.

Sin embargo, este trabajo en equipo es la principal fuente de debilidades, ya que el trabajo de los alumnos depende de los resultados del trabajo de sus compañeros, por lo que en muchas ocasiones el trabajo de un alumno queda paralizado, bien porque otro compañero no ha realizado su trabajo a tiempo o bien porque no hay la suficiente comunicación entre ellos. Así, el trabajo global se retrasa en el tiempo innecesariamente. De hecho, la falta de comunicación fue el problema que más frecuentemente encontraron los tutores, incluso entre alumnos que habían solicitado el TFG de forma conjunta.

Por otra parte, el trabajo se planteó a los alumnos como un trabajo que iba a ser guiado por los profesores y en el que se iban a desarrollar una serie de talleres impartidos por los 


\section{F.J. Camacho Torregrosa, A.M. Pérez Zuriaga, H. Coll Carrillo, J. Alcalá González, I. Romero Gil y M.E. Garrido de la Torre}

profesores de cada especialidad para consolidar conceptos ya adquiridos durante el grado, ampliarlos y enseñar cómo utilizar determinadas herramientas informáticas. Esta puede ser la causa por la que un gran porcentaje de los alumnos no ha mostrado una actitud proactiva, sino más bien ha consultado a los profesores cada uno de los pasos a dar, no siendo esta la idea de un TFG.

Adicionalmente, hay que tener en cuenta que en este proyecto ha participado un número considerable de alumnos con marcadas diferencias entre ellos: diferentes titulaciones (GIC y GIOP), diferentes asignaturas pendientes, diferentes expedientes, diferentes horarios. Todo ello ha provocado que se hayan planteado problemas a la hora del trabajo en equipo, especialmente a la hora de fijar un horario en el que pudieran asistir todos los participantes. A lo largo del semestre se ha reservado un aula todos los viernes de 12:15 a 17:15 horas y, únicamente, en dos ocasiones se contaba con la presencia de todos los alumnos.

Una situación similar se ha observado con los profesores, aunque esta se ha resuelto en general gracias a que en las disciplinas con más demanda se contaba con dos profesores.

\subsection{Fortalezas}

Como se ha citado anteriormente, el trabajo se ha planteado como un trabajo multidisciplinar, pero además se ha planteado para tres alternativas de una variante, es decir hay alumnos que deben llevar a cabo un trabajo muy similar pero particularizado para cada una de las alternativas. De esta forma, los alumnos pueden trabajar en equipo, colaborarando entre ellos y resolver cuestiones dentro de una misma área de conocimiento, y no sólo entre las diferentes disciplinas.

Asimismo, esta situación conlleva también una economía de escala para los profesores, ya que un mismo seminario (con sus correspondientes enseñanzas y aclaraciones) es válido para varios alumnos. Esto supone una ventaja sobre todo al inicio del trabajo, cuando el nivel de todos los alumnos es similar. A medida que avanza el desarrollo del trabajo, cada alumno también se desarrolla de forma diferente, por lo que las tutorías individuales pasan a reemplazar en gran medida a los seminarios.

Estos seminarios facilitan el trabajo de los alumnos y evitan que pierdan tiempo y retrasen su trabajo buscando cómo llevarlo a cabo. No obstante, hay que tener especial cuidado en este aspecto ya que puede conllevar una actitud pasiva por parte de los alumnos.

Por otra parte, ya se ha explicado en el apartado correspondiente que los alumnos han sido repartidos entre las distintas alternativas considerando su expediente y su situación académica. De esta forma, se ha intentado que los alumnos dentro de una misma alternativa tengan el mismo ritmo de trabajo y que la falta de resultados de un miembro del equipo no penalice en exceso al resto. Esto se ha revelado como un acierto, pues los conflictos intragrupo han sido mucho menos frecuentes que en otros cursos.

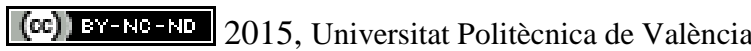

Congreso In-Red (2015) 
Desarrollo de un Trabajo de Fin de Grado Multidisciplinar en Ingeniería Civil como preparación del alumno para la vida profesional

Finalmente, remarcar que los seminarios pueden ser aprovechados parcialmente para conformar objetos de aprendizaje, lo que facilitará el desarrollo de futuros TFGs.

\subsection{Propuestas}

Considerando la experiencia vivida a lo largo del presente semestre en el que se ha desarrollado el TFG multidisciplinar se proponen las siguientes actuaciones que pueden facilitar el trabajo de otros profesores que quieran desarrollar un proyecto similar:

- Contar con profesores que estén verdaderamente involucrados en el proyecto, ya que, especialmente, el primer año conlleva un trabajo adicional considerable por parte de los profesores.

- Fijar en los calendarios desarrollados por la ERT dos horas a la semana dedicadas exclusivamente al TFG, tanto en los calendarios de cuarto curso como los de tercero, con el fin de facilitar las reuniones de grupo. De esta forma, se consideraría el TFG como lo que realmente es: una asignatura.

- Proponer el TFG a los alumnos y publicar la asignación de trabajos antes de la fecha límite para solicitar un TFG de la subasta de la Escuela. De esta forma, se aumenta el número de solicitantes que de otra forma prefieren recurrir directamente a la subasta para evitar quedarse sin tutor.

- Distribuir a los alumnos en grupos según la nota media de su expediente y según su situación académica, especialmente en cuanto a lo que asignaturas pendientes se refiere.

- Realizar una entrevista personal a los alumnos, evaluando su capacidad y, especialmente, el interés y la proactividad. Esta entrevista debería ser determinante para escoger al alumno para realizar el TFG.

- Asignar los TFG cuyos resultados sean más determinantes a los alumnos con más disponibilidad, es decir, con menos asignaturas pendientes.

- Estudiar en profundidad el calendario y el contenido, tanto de los talleres como de las tutorías grupales y seminarios.

- Establecer con claridad a los alumnos el contenido global del proyecto multidisciplinar y de su TFG en particular.

- Fijar un calendario de hitos para los alumnos, con el fin de que el trabajo no se retrase en exceso y, sobre todo, de que el trabajo de un alumno no se vea perjudicado por el retraso de otro de sus compañeros.

- Evaluar las competencias transversales al comenzar el TFG y tras su presentación.

- Dentro del primer mes, los alumnos de cada alternativa deberían definir un compañero como líder del equipo, con el fin de detectar los problemas anteriormente mencionados sin necesidad de que lo haga alguno de los tutores.

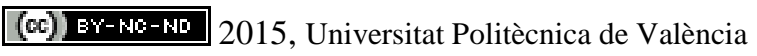

Congreso IN-RED (2015) 
Con todo ello, se considera que un proyecto similar puede llevar resultados más que aceptables, aunque considerando el elevado número de alumnos y de profesores, siempre puede haber imprevistos.

\section{Conclusiones}

A lo largo del presente documento se ha expuesto una nueva metodología para el desarrollo de Trabajos Fin de Grado que permite tanto que los alumnos logren un alto grado de especialización en las materias elegidas como que desarrollen las competencias transversales de trabajo en equipo y liderazgo.

Los resultados provisionales que se están observando han permitido ver que, si bien es necesario pulir algunos aspectos, el desarrollo del trabajo ha sido un éxito, extrapolable a otras disciplinas.

En un futuro debería ser incluso posible la organización de TFGs incluyendo alumnos de otras Escuelas, permitiendo un mayor grado de multidisciplinariedad.

\section{Referencias}

BARKLEY, E.; CROSS, D.P. Y MAJOR, C. H. Técnicas de aprendizaje colaborativo. Ediciones Morata

UPV. Dimensiones Competenciales

http://www.upv.es/contenidos/ICEP/info/DimensionesCompetenciales.pdf (Consulta 30/5/2015)

UPV. Normativa Marco de Trabajos Fin de Grado y Fin de Máster.

http://www.upv.es/entidades/SA/mastersoficiales/U0594312.pdf (Consulta 30/5/2015)

(cc) EY-NC-ND 2015, Universitat Politècnica de València

Congreso In-Red (2015) 